\title{
An Efficient Filtering Technique for Denoising Colour Images
}

\author{
K. Arun Sai, K. Ravi \\ Department of Electronics and Communication Engineering, Institute of Aeronautical Engineering, India
}

\begin{tabular}{l} 
Article Info \\
\hline Article history: \\
Received Apr 16, 2018 \\
Revised Jul 10, 2018 \\
Accepted Jul 16, 2018 \\
\hline
\end{tabular}

\section{Keyword:}

Color filter array

Gradient filter out noise

Interpolation

Signal to noise ratio

\begin{abstract}
Single-sensor digital cameras capture image with the aid of masking the sensor surface along a colour filter array(CFA) such that every sensor pixel solely samples certain of three primary colour values i.e., R (red), $\mathrm{G}$ (green) and B (blue). To get a full-colour image, an interpolation method commonly referred in conformity with CFA demosaicking is required to estimate the other two contributions for producing a full-colour image. But, the clutter in imaging sensors not only corrupts the colour filter array but also introduces artifacts at some stage in the colour interpolation step and affects the characteristics of image. To acquire high quality full-colour image, a kind of viable and effective interpolation algorithm based over gradient is used. This technique can remove the noise effectively by retaining image border and detail data clearly.
\end{abstract}

Copyright $@ 2018$ Institute of Advanced Engineering and Science. All rights reserved.

Corresponding Author:

K. Arun Sai,

Department of Electronics and Communication Engineering,

Institute of Aeronautical Engineering,

Dundigal Hyderabad, Telangana-500043, India.

Email: arunsai.k4@gmail.com

\section{INTRODUCTION}

With the evolution of science and technology in the defence and civil sectors, the colour camera with single CCD are extensively used as image input device. The colour image from the single CCD digital camera is referred to as the CFA (colour filter array) image. Currently, the colour image recovery algorithm primarily based on the CFA is widely used. The present writing put forward a lot over colour interpolation algorithms, namely adaptive interpolation technique [1], weight coefficient technique [2], interactive interpolation method [3], based on vector [4], and so on in an optimized way. However often used interpolation technique is bilinear interpolation technique [5] that belongs to the single channel independent interpolation method. In this technique the unknown colour factor among a point is computed generally by means of the average of adjacent same colour components. This approach runs faster, however ignores the detail data and the correlation between the three-colour channels, therefore the bilinear method frequently cannot achieve effective interpolation. Colour proportion constant method [6], it has an intense relation within different colour channels considering the correlation and the quality of the reconstructed image, was improved, but in fact still belongs to the class concerning bilinear method. The method primarily based on gradient [7], [8], researchers introduced the interpolation algorithm based totally on gradient, that can select the appropriate interpolation direction and can avoid the appearance of the zigzag pattern in the edge of recovered image. But, this approach does no longer consider the influence over noises of the image and accomplish the colour recovery distortion close by the noise. The proposed interpolation method can remove the clutter primarily based on gradient and effectively excerpt the impact of the noise by retaining the edge and the detail information of the image.

Removal of noise in color image in an optimized way is achieved by using red component for the interpolation. Red component is used for interpolation from the R, G, and B components for removing the noise in order to optimize the computations required. As $\mathrm{R}$ and $\mathrm{B}$ components are accounted each as $1 / 4 \mathrm{Of}$ the 
total number of the pixels in Bayer CFA pattern. Whereas the $G$ component is $1 / 2$ of the total number of pixels. Using $\mathrm{G}$ component for the interpolation, in order to remove noise takes more time as it includes more computations compared to $\mathrm{R}$ and $\mathrm{B}$ component.

\section{CFA IMAGE COLOR RECOVERY METHOD}

\subsection{CFA (color filter array) image}

There is only colour component gray value on each lattice point in the CFA (colour filter array) image. Because of the human eye photosensitive characteristic, at present the GRGB colour swatches is most commonly used, namely Bayer colour filter array, as shown in Figure 1.

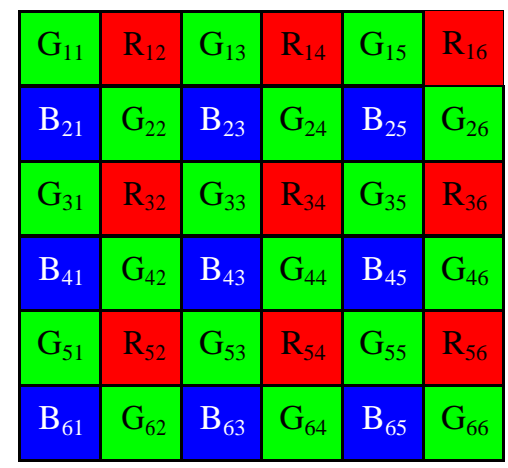

Figure 1. Bayer CFA pattern

It uses a group of red and green filter or a group of blue and green filter by turns to obtain image, the number of green pixels are half part over the other pixels, and the red and blue then each for 1/4. Due to the green component accounted for half of the total, hence it has more detail information over image, therefore, the interpolation algorithm begins mostly advance from restoring $\mathrm{G}$ component.

\subsection{Filter out noise method based on gradient}

The technique based on gradient, does not consider the impact of noise to algorithm, then the image entails G11 noise, if the clutter as colour information involved in calculation after recovering image, not only makes the colour distortion, but also using the information of four restore point close to the noise, their colour component also can appear distortion. Hypothesis, Gi,j, is a high frequency clutter point, then, G 1-i,j $\mathrm{G} 1+\mathrm{i}, \mathrm{j}, \mathrm{Gi}, \mathrm{j}-1 \mathrm{Gi}, \mathrm{j}+1$ and $\mathrm{Gi}, \mathrm{j}$, their $\mathrm{G}$ factor will appear distortion. Therefore, it is important to remove clutter for getting better colour image, but generally the median and mean filter is used for the gray image method, are not appropriate for CFA distribute images. This paper study two techniques primarily based on gradient form Hibbard [3] and Laroche [4], through the gradient of the calculation results in both technique connected, eliminating the impact of the noise.

As shown in Figure $1, B_{i, j}$, is a point of B component in the image, in order to restore $G_{i j}, G_{i j}$, says the value of $\mathrm{G}$ component in this point. In (1) A1 is horizontal internal gradient and B1 is vertical internal gradient, through calculate one order differential such as formula (1).

$$
\left\{\begin{array}{l}
A_{1}=\left|G_{i, j-1}-G_{i, j+1}\right| \\
B_{1}=\left|G_{i-1, j}-G_{i+1, j}\right|
\end{array}\right.
$$

In (2) A2 is horizontal external gradient, B2 is vertical external gradient, through calculation two order differential such as formula (2).

$$
\left\{\begin{array}{l}
A_{2}=\left|2 \times B_{i, j}-B_{i, j-2}-B_{i, j+2}\right| \\
B_{2}=\left|2 \times B_{i, j}-B_{i-2, j}-B_{i+2, j}\right|
\end{array}\right.
$$

According to the gradient results of internal and external two layers, to locate edge information of image if really exist, or have the influence of the noise point. Set TH is enumeration variable, for being 
clutter that in the up and down or so said at the point of $\mathrm{G}_{\mathrm{ij}}$, and for having horizontal or vertical edge information in this point. To compute the formula concerning TH.

$$
T H= \begin{cases}\text { up } & \left(A_{1}<B_{1}\right) \&\left(A_{2}=B_{2}\right) \&\left(\max \left|2 \times G_{i, y}-G_{i-1, j}-G_{i+1, j}\right|=j-1\right) \\ \text { down } & \left(A_{1}<B_{1}\right) \&\left(A_{2}=B_{2}\right) \&\left(\max \left|2 \times G_{i, y}-G_{i-1, y}-G_{i+1, j}\right|=j+1\right) \\ \text { left } & \left(A_{1}>B_{1}\right) \&\left(A_{2}=B_{2}\right) \&\left(\max \left|2 \times G_{x, j}-G_{i, j-1}-G_{i, j+1}\right|=i-1\right) \\ \text { right } & \left(A_{1}>B_{1}\right) \&\left(A_{2}=B_{2}\right) \&\left(\max \left|2 \times G_{x, j}-G_{i, j-1}-G_{i, j+1}\right|=i+1\right) \\ \text { no } & \left(A_{1}=B_{1}\right) \&\left(A_{2}=B_{2}\right) \\ \text { level } & \left(A_{1}>B_{1}\right) \&\left(A_{2}>B_{2}\right) \\ \text { erect } & \left(A_{1}<B_{1}\right) \&\left(A_{2}<B_{2}\right) \\ & (i=2,4,6 \ldots ; j=1,3,5 \ldots ; x=i-1, i+1 ; y=j-1, j+1)\end{cases}
$$

In (3) $\max \left|2 \times G_{i}, y-G_{i-1, j}-G_{i+1, j}\right|=j+1$ is the column location of point, whose distance is farthest between $G_{i-1, j}$ and $G_{i+1, j}$, max $\left|2 \times G_{x}, j-G_{i, j-1}-G_{i, j+1}\right|$ whose distance is farthest between, $G_{i, j-1}$ and $G_{i, j+1}$, If $T H$ equals up then it indicates, $G_{i, j-1}$ is the noise, down indicates, $G_{i, j+1}$ is the noise, left indicates $G_{i-1, j}$, is the noise right indicates $G_{i+1, j}$ is the noise, no indicates that there is no noise and no edge, level indicates that there is an edge in vertical direction, erect indicates that there is an edge in horizontal direction. So the finally calculate formula of $\mathrm{G}_{\mathrm{i}, \mathrm{j}}$, like formula (4) below.

$$
G_{i, j}= \begin{cases}\left(G_{i-1, j}+G_{i+1, j}+G_{i, j+1}\right) / 3 & \text { TH }=\text { up } \\ \left(G_{i-1}+G_{i+1, j}+G_{i, j-1}\right) / 3 & T H=\text { down } \\ \left(G_{i+1, j}+G_{i, j-1}+G_{i, j+1}\right) / 3 & \text { TH }=\text { left } \\ \left(G_{i-1, j}+G_{i, j-1}+G_{i, j+1}\right) / 3 & \text { TH }=\text { right } \\ \left(G_{i-1, j}+G_{i+1, j}+G_{i, j-1}+G_{i, j+1}\right) / 4 & \text { TH }=\text { no } \\ \left(G_{i-1, j}+G_{i+, j}\right) / 2 & \text { TH }=\text { level } \\ \left(G_{i, j-1}+G_{i+, j+1}\right) / 2 & \text { TH = erect }\end{cases}
$$

The noise removal after the $\mathrm{G}_{\mathrm{ij}}$ restored is as follows,

$$
\left\{\begin{aligned}
G_{i, j-1} & =G_{i, j} & & \text { TH }=\text { up } \\
G_{i, j+1} & =G_{i, j} & & \text { TH }=\text { down } \\
G_{i-1, j} & =G_{i, j} & & \text { TH }=\text { left } \\
G_{i+1, j} & =G_{i, j} & & \text { TH }=\text { right }
\end{aligned}\right.
$$

\section{RESULTS}

The test image which is used to apply the denoising technique is of the size $925(\mathrm{H}) \times 590(\mathrm{~V})$. The Figure 2. is the original CFA image with noise and Figure 3. is the denoised image effectively filter the noise and make the image look more refined.

The quality of two images is measured using SNR. Here Figure 2 is the image before applying the filter technique and Figure 3 is the image after applying the filtering technique. 


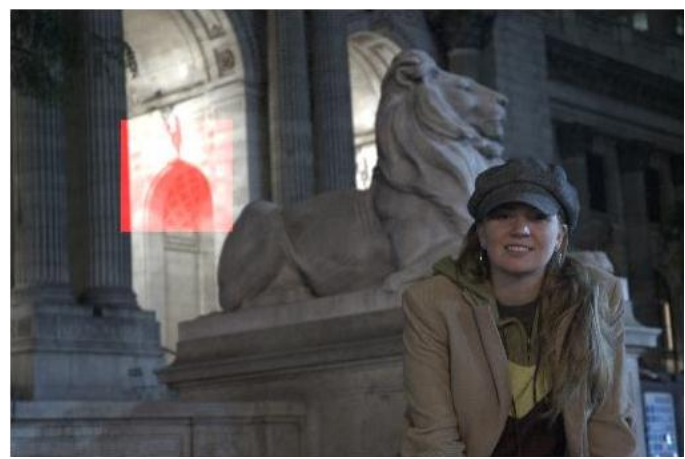

Figure 2. CFA image with noise

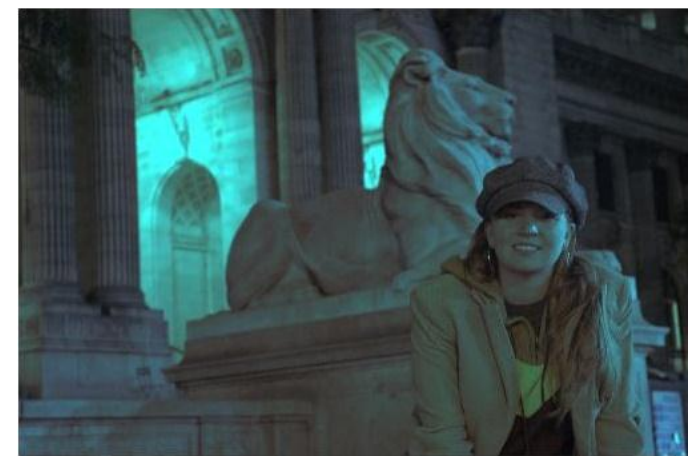

Figure 3. Denoised image after applying the algorithm

SNR for a given image can be computed using the expression,

$$
\mathrm{SNR}=\mu / \sqrt{ } \mathrm{LSD}^{2}
$$

Where $\mu$ is average gray of colour image and $\mathrm{LSD}_{\max }$ is local variance maximum.

Table 1 shows the SNR of two Figures.

Table 1. SNR of two Figures

\begin{tabular}{ccc}
\hline Figure & SNR (existing) & SNR (proposed) \\
\hline 2 & 17.09333 & 33.165210 \\
3 & 21.66333 & 39.215886 \\
\hline
\end{tabular}

\section{CONCLUSION}

The CFA image colour interpolation method introduced in this paper used filter out noise interpolation method based on gradient in an optimized way to avoid noise on the colour recovery influence. This method has wide application in defence and civil sectors which improves the signal to noise ratio of a colour image and has a wide application prospect.

\section{REFERENCES}

[1] Haijiang Sun and Yanjie Wang, "Colour Filtering Method for CFA Images Based on Gradient", International Conference on Communication Systems and Network Technologies, 2012.

[2] J. E. Adams, "Design of Practical Colour Filter Array Interpolation Algorithms for Digital Cameras", IEEE, Image Processing, Chicago, vol. 1, pp. 488-492, 1998.

[3] Pala Mahesh Kumar, "Satellite Image Denoising using Local Spayed and Optimized Center Pixel Weights", International Journal of Electrical and Computer Engineering (IJECE), vol. 4, no. 5, pp. 751-757, 2014.

[4] Jan Aelterman, et al, "Locally Adaptive Complex wavelet-based Demosaicing for colour filter array Images", SPIE Wavelet Applications in Industrial Processing VI, San Jose. CA. USA, January 2009, vol. 7248, pp. 72480j1-72480j12.

[5] B. K. Gunturk, et al, "Colourplane Interpolation using Alternating Projections", IEEE Transactions on Image Processing. Atlanta, 2002, vol. 11, no. 9, pp. 997-1013.

[6] B. K. Gunturk, et al, "Demosaicking: Colour Filter Array Interpolation", IEEE, Signal Processing Magazine, January 2005.

[7] R. G. Keys, et al, "Cubic Convolution Interpolation for Digital Image Processing", IEEE Transactions on Acoustic, Speech and Signal Processing, Tulsa, 1981, vol. 29, pp. 1153-1160.

[8] Soo-Chang Pei, "Effective Colour Interpolation in CCD Colour Filter Arrays Using Signal Correlation", IEEE Transactions On Circuits And Systems For Video Technology, vol. 13, no. 6, pp. 503-513, 2003.

[9] R. H. Hibbard, "Apparatus and Method for Adaptively Interpolating a full colour Image Utilizing Luminance Gradients", U.S, Patent 5, 382, 976, 1995.

[10] C. A. Laroche and M. A. Prescott, "Apparatus and Method for Adaptively Interpolating a full colour Image Utilizing Chrominance Gradients”, U.S, Patent 5, 373, 322, Dec. 1994.

[11] BASLER A.201bc User's Manual. www.basler-vc.com, 2005. 


\section{BIOGRAPHIES OF AUTHORS}

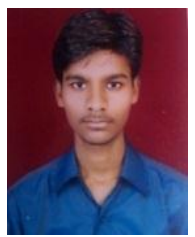

K. Arun Sai received the Bachelor's degree in Technology (Electronics and Communication Engineering) from MLR Institute of Technology (JNTUH) Hyderabad, Telangana, India in 2011, and the Master's degree in Technology (Digital Electronics and Communication Systems) from Sri Indu College of Engineering and Technology (JNTUH) Hyderabad, Telangana, India in 2013. He is currently working as Assistant Professor in Institute of Aeronautical Engineering, Dundigal, Hyderabad, India.

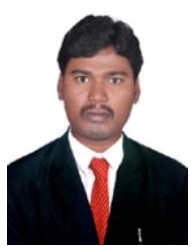

K. Ravi received the Bachelor's degree in Technology (Electronics and Communication Engineering) from SRTIST, Nalgonda (JNTUH), Telangana, India, in 2008, and the Master's degree in Technology (Microelectronics and VLSI Design) from NIT Calicut, Kerala, India in 2011. He is currently working as Assistant Professor in Institute of Aeronautical Engineering, Dundigal, Hyderabad, India. 